\title{
Estimating the risk of HIV transmission from homosexual men receiving treatment to their HIV-uninfected partners
}

\author{
Timothy B Hallett, ${ }^{1}$ Colette Smit, ${ }^{2}$ Geoff P Garnett, ${ }^{1}$ Frank de Wolf ${ }^{1,2,3}$
}

- Supplementary figures and appendix are published online only. To view these files please visit the journal online (http://sti. bmj.com).

'Department of Infectious Disease Epidemiology, Imperial College London, UK ${ }^{2}$ HIV Monitoring Foundation, Amsterdam, Netherlands ${ }^{3}$ Academic Medical Centre, University of Amsterdam, The Netherlands

\section{Correspondence to} Dr Timothy B Hallett, Department of Infectious Disease Epidemiology, Imperial College London, St Mary's Campus, Norfolk Place, London W2 1PG, UK:

timothy.hallett@imperial.ac.uk

TBH and CS contributed equally to this paper.

Accepted 20 May 2010

Published Online First

18 July 2010

\section{ABSTRACT}

Objective To determine how the risk of HIV transmission from homosexual men receiving antiretroviral treatment is related to patterns of patient monitoring and condom use.

Methods A stochastic mathematical simulation model was developed of cohorts of men in the Netherlands who have sex with men (MSM), defining the parameters of the model using observational cohort data. The model incorporates viral load trends during first-line treatment, patient monitoring and different scenarios for the way in which condom use may depend on recent viral load measurements. The model does not include the effect of sexually transmitted infections on HIV transmission. Results For MSM receiving treatment, the risk of transmitting HIV to their long-term partner is $22 \%$ (uncertainty interval: 9-37\%) if condoms are never used. With incomplete use (in 30\% of sex acts) the risk is reduced slightly, to $17 \%(7-29 \%)$. However, the risk is as low as $3 \%(0.2-8 \%)$ when men receiving treatment use condoms only 6 months beyond their last undetectable viral load measurement. The risk is further reduced when 3 months is the time period beyond which condoms are used

Conclusions When condom use by HIV-infected men receiving combination treatment with antiretroviral agents is based on their last viral load measurement, the transmission risk is much lower than with incomplete condom use. The key message for patients is that although always using condoms during treatment is the best way to protect partners from the risk of HIV transmission, when such use cannot be achieved, the second best strategy is to use condoms whenever the last undetectable viral load was measured more than 3 months ago.

\section{INTRODUCTION}

Since the mid-1990s, people infected with HIV in industrialised countries have been able to access combination antiretroviral treatment (cART), ${ }^{1}{ }^{2}$ which has substantially decreased HIV-associated mortality in these populations. ${ }^{3}$ More recently, this trend has been repeated in many developing countries, along with signs of decreasing HIV mortality. ${ }^{4}$ In both settings, there is a trend for earlier initiation of treatment ${ }^{5}$ and increasing debate about whether starting treatment earlier (or even immediately on HIV diagnosis) is advisable in order to improve clinical outcomes and reduce HIV transmission. ${ }^{6} 7$ As the population receiving treatment grows its potential contribution to HIV epidemic increases. Therefore, we expect that the infectiousness and the sexual behaviour of those receiving treatment will become key determinants of the trajectory of the global epidemic in the coming years.

The rate of HIV transmission from infected people is closely tied to their plasma viral load, which cART can reduce to very low levels. ${ }^{8}$ On this evidence, in 2008 the Swiss National AIDS Commission (EKAF) suggested that there was effectively no risk of sexual transmission from patients receiving cART, provided that they (i) follow the guidelines for antiretroviral treatment strictly, (ii) have no detectable viral load when measured in the past 6 months and (iii) have no other sexually transmitted infection (STI). ${ }^{9}{ }^{10}$ The implication that such patients need not use condoms with their sexual partners was warmly welcomed by patient groups and activists. ${ }^{11}$

In response, many argued that this was not a helpful public health message. Although the chance of transmission from men receiving treatment is likely to be low, it is unlikely to be zero, since at least one transmission event from a man receiving successful cART has occurred, ${ }^{12}$ and over many sex acts among many men, a small rate of transmission could translate into a large number of new infections. ${ }^{13} 14$ A simple model was used to argue this point, ${ }^{13}$ but it did not estimate the absolute risk of transmission from men receiving treatment; nor did it account for condom use being conditional on a recent viral load measurement, as the EKAF statement recommended. ${ }^{9}$

In this paper, using uniquely detailed data on the trends in viral loads among men receiving treatment ${ }^{17}$ and a new stochastic mathematical model, we estimate the risk of HIV transmission from men in the Netherlands who have sex with men (MSM) and determine how this is influenced by various patterns of condom use (including those based on the Swiss recommendations) and schedules of viral load monitoring.

\section{METHODS}

Following earlier work, ${ }^{18}{ }^{19}$ we developed a stochastic individual-based simulation model of viral load trends, HIV transmission risk and patient monitoring among cohorts of MSM in the Netherlands. The vast range of possibilities for the evolution of viral load over time was conceptualised as three distinct trajectories (figure 1). Data from the ATHENA observational cohort of HIV-infected individuals in the Netherlands ${ }^{17}$ were used to evaluate the parameters (table 1, with further detail provided in the online technical appendix).

The risk of transmission is characterised as the probability that a man receiving treatment will 


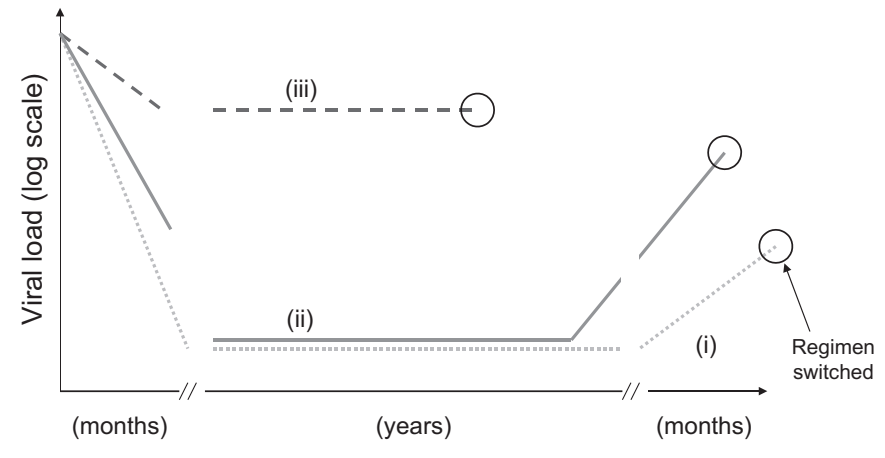

Time since HIV infection

Figure 1 Schematic representation of the three assumed trajectories of (log) viral load after treatment initiation: (i) suppression achieved and adherence good (dotted line); (ii) suppression achieved but adherence poor (solid line); and (iii) suppression not achieved (dashed line). The circles indicate when the regimen was changed. Parameter values are presented in table 1 and details are provided in the online supplementary appendix.

infect his uninfected sexual partner over the course of first-line treatment. We examined only the special case where a partnership is maintained over the entire duration of first-line treatment and did not include the effect of STIs on the chance of HIV transmission. In most analyses, the functional relationship between plasma viral load and the chance of transmission in each unprotected sex act was based on a Hill function fitted to observational data on HIV transmission in Zambia by Fraser et al. ${ }^{20}$ This function is supported by recent review of transmission rates ${ }^{15} 16$ and is denoted the 'Fraser et al' assumption. The function was rescaled to match the observed approximate transmission risk for each sex act for MSM ( $\sim 0.01$ for men with log-viral loads of $\sim 4.5$ log-copies) ${ }^{13}$ (supplementary figure S2). An alternative assumption for the relationship (linear trend in log-risk and log-viral load ${ }^{21}$ : see figure S2) was used as well in sensitivity analyses: this is denoted the 'Wilson et al' assumption. We assumed that there were 100 sex acts a year in the partnership and that the efficacy of condoms in reducing transmission is $95 \%$. In uncertainty analyses, the model assumption for which there was least directly relevant data was varied independently in a Monte Carlo analysis: specifically, the frequency of sex acts per years (uniform between 50 and 150), the efficacy of condoms when used (uniform between $85 \%$ and $100 \%$ ) and chance of transmission per sex act without treatment (uniform from 0.0075 to 0.0125 ). A combined $95 \%$ uncertainty interval (the range between the 2.5th and 97.5th centiles of the resultant distribution) was calculated.

In the model, reflecting clinical guidelines, ${ }^{22}$ patients with undetectable viral loads are monitored every 4 months. When viral load is detectable, the patient returns for another appointment after 8 weeks and the treatment regimen is then changed if retesting confirms the finding.

Various scenarios for condom use in partnerships were considered: (i) never using condoms; (ii) using condoms in $30 \%$ of sex acts ${ }^{24}$; (iii) not using condoms if viral load was undetectable at the last measurement in the past 6 months; and, (iv) always using condoms. Scenario (iii) corresponds to the way that the EKAF statement has been interpreted and we considered two variants whereby the decision not to use condoms is based on the last measurement in the past 3 months (instead of 6 months) or the last viral load measurement ever taken (ie, irrespective of time).

\section{RESULTS}

We found that men receiving treatment pose a substantial risk of HIV transmission (22\% (9-37\% in uncertainty analysis)) to their partners if they do not use condoms (figure 2), and the relationship between viral load and transmission is as described by Fraser et al. ${ }^{20}$ This risk is generated in three ways: (i) treatment is not sufficient to suppress viral load, so transmission can occur even if the regimen is quickly changed; (ii) the level of virus can rebound quickly and reach high levels before detection and change of regimen; (iii) even with suppressed viral loads, the risk of transmission is not zero, so that over the many sex acts during treatment, the cumulative chance of transmission becomes non-negligible.

Using condoms $30 \%$ of the time reduces the chance of transmission but only marginally (to $17 \% \quad(7-29 \%$ in

Table 1 Natural history parameters for HIV infection receiving first-line treatment (Source: ATHENA cohort data ${ }^{17}$ ) Refer to the technical appendix for details

\begin{tabular}{|c|c|c|c|}
\hline Parameter & & Value & Unit \\
\hline \multirow[t]{2}{*}{$\log _{10}$ viral load at treatment initiation (normal distribution) } & Mean & 5.1 & \multirow[t]{2}{*}{ Log copies per microlitre } \\
\hline & Variance & 0.40 & \\
\hline Fraction achieving suppression* & & 0.70 & Fraction \\
\hline Fraction with good adherence $†$ & & 0.94 & Fraction \\
\hline Waiting time until viral suppression, if suppression and good adherence (exponential distribution) & Median & 5.38 & Months \\
\hline Waiting time until viral suppression, if suppression and not good adherence (exponential distribution) & Median & 5.92 & Months \\
\hline \multirow{5}{*}{$\begin{array}{l}\text { Waiting time (months) until viral rebound, if good adherence (double Weibull distribution), assuming } \\
\text { shortened (s) survival times (used unless otherwise stated) or extended (e) survival. }\end{array}$} & Shape $_{1}$ & 1.14 (e) 10.23 (s) & \multirow[t]{5}{*}{ Months } \\
\hline & Scale $_{1}$ & 456.65 (e) 153.25 (s) & \\
\hline & Shape $_{2}$ & 1.92 (e) 1.25 (s) & \\
\hline & Scale $_{2}$ & 20.70 (e) 41.58 (s) & \\
\hline & Weighting & 0.91 (e) 0.77 (s) & \\
\hline \multirow[t]{2}{*}{ Waiting time until viral rebound, if not good adherence (Weibull distribution) } & Shape $_{1}$ & 1.14 & \multirow[t]{2}{*}{ Months } \\
\hline & Scale $_{1}$ & 132.86 & \\
\hline Rate of increase in $\log _{10}$ viral load on rebound, if good adherence $\neq$ & Rate & $0.07(\mathrm{se}=0.002)$ & $\log _{10}$ copies per microlitre per month \\
\hline Rate of increase in $\log _{10}$ viral load on rebound, if not good adherence $\neq$ & Rate & $0.16(\mathrm{se}=0.01)$ & $\log _{10}$ copies per microlitre per month \\
\hline Maximum survival time with non-suppressed viral load & Max & 1 & Years \\
\hline \multirow[t]{2}{*}{$\log _{10}$ viral load if no suppression (normal distribution) } & Mean & 3.80 & \multirow[t]{2}{*}{ Log copies per microlitre } \\
\hline & Variance & 0.84 & \\
\hline
\end{tabular}

${ }^{*}$ Suppression is defined as at least two consecutive viral load measurements $<500$ HIV RNA copies per millilitre.

†Adherence is classified as good or poor based on the measurement of plasma drug levels.

$\ddagger$ At all times in the simulation, viral load is limited to less than or equal to the 95th centile of viral loads at treatment initiation in the ATHENA data (equal to six log-copies). 


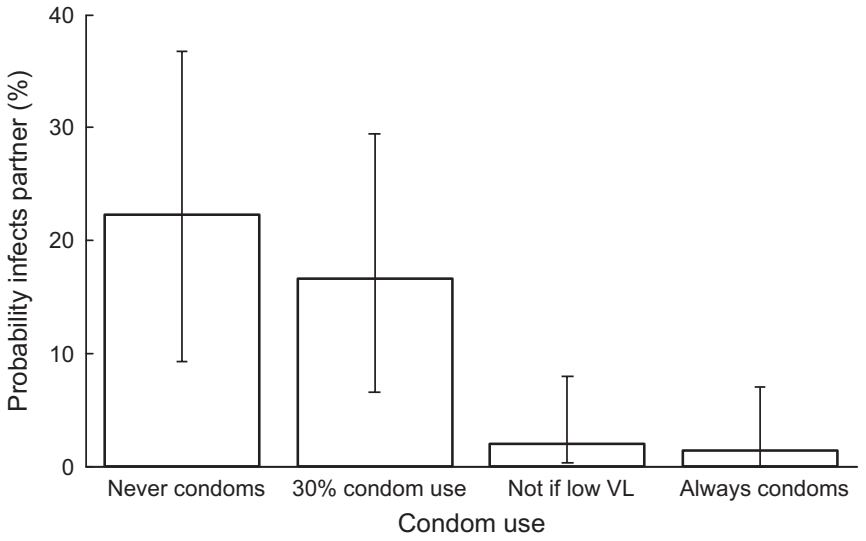

Figure 2 Probability of transmission to partner during first-line treatment, if condoms are never used; condoms are used $30 \%$ of the time; (iii) condoms are used unless last viral load (VL) measurement in the past 6 months was undetectable; or always using condoms. Errorbars show the $95 \%$ uncertainty interval, as described in the text. It is assumed that the partnership is maintained over the entire course of first-line treatment and that viral load is related to transmission rate, as Fraser et $a l^{20}$ have described.

uncertainty analysis)), since there remains a substantial numbers of unprotected sex acts overall. In contrast, men who always use condoms unless their viral load was undetectable at the last measurement in the past 6 months are much less likely to transmit HIV to their partner (chance of transmission: $3 \%$ $(0.2-8 \%$ in uncertainty analyses)). This is because the risk generated in the first two ways has been largely removed. Men following this strategy, on average, use condoms $10 \%$ of the time they are receiving treatment, while reducing the risk of transmission to $3 \%$. The risk with this strategy is nevertheless greater than always using condoms, in which the chance is $1 \%$ $(0-7 \%$ in uncertainty analysis) (figure 2$)$. The risk is not zero, because condom efficacy is not perfect.

Using the alternative ('Wilson et al') assumption about the relationship between plasma viral load and transmissibility, the overall chance of infection if condoms are not used is higher (47\% vs $22 \%$ ), but the same key result is reproduced: men who use condoms unless their viral load was undetectable in the past 6 months are much less likely to transmit HIV to their partner than if condoms are used intermittently (online figure S3).

The frequency with which patients receiving treatment are monitored is a key determinant of the chance of HIV transmission (figure 3A). Patients monitored frequently can be quickly switched to new regimens if first-line treatment fails-that is, before viral load increases and they have exposed their partner to an increased risk of transmission for a long period. The second effect of increased frequency of monitoring is the reduction in overall condom use. This is because with less frequent monitoring, many men with suppressed viral loads would use condoms since they have not had a recent viral load measurement. With monitoring every 18 months, condoms would be used in $\sim 70 \%$ of sex acts, but with monitoring every 3 months, condoms are used in only $9 \%$.

The benefit of patient monitoring is influenced by the proportion of patients lost to follow-up. The probability of infecting a partner increases from $2 \%$ when none of the patients are lost to follow-up, to $5 \%$ when $20 \%$ do not return for care (figure $3 \mathrm{~B}$ ). This is because increases in viral load go undetected for longer, exposing partners to a higher risk of infection.

Finally, we examined three ways in which condom use could be based on viral load measurements (online figure S5). The
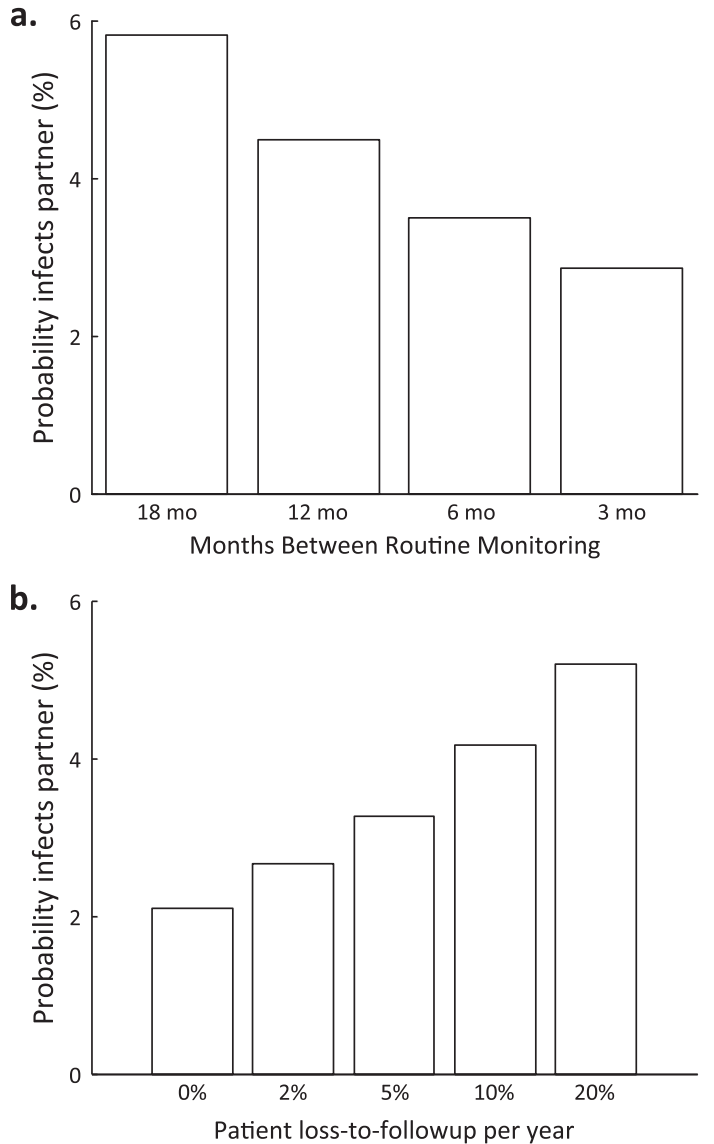

Figure 3 The influence of $(A)$ monitoring frequency and $(B)$ loss to follow-up on the probability of HIV transmission, assuming condoms are used unless last viral load measurement in the past 6 months was undetectable. It is assumed that the partnership is maintained over the entire course of first-line treatment and that viral load is related to transmission rate, as Fraser et $a l^{20}$ have described.

decision not to use condoms could be based on an undetectable viral load in the past 3 months, the past 6 months or the last measurement ever. With the decision based on a measurement in the past 3 months, there is reduced transmission compared with a decision based on the past 6 months, provided that patients are monitored at least every 3-12 months. However, with the decision based only on the last measurement regardless of time, the chance of transmission is higher, especially if monitoring intervals are longer.

\section{DISCUSSION}

The debate about the EKAF statement on HIV transmission from patients receiving $\mathrm{CART}^{13}$ and earlier modelling work ${ }^{14}$ did not consider the way in which deciding not to use condoms might be conditional on the last viral load measurement. There was also little focus on estimating the rate of HIV transmission across the population or exploring how it is influenced by patterns of patient monitoring. In this paper we have shown that basing the decision to use condoms on viral load provides substantially better protection to partners than incomplete condom use, provided that the measurement is within the past 3-6 months. Compared with always using condoms, the viral-load-dependent strategy allows slightly more HIV transmission (2-3\% vs $1 \%$ ). However, as condoms are needed much less of the time (10\% vs $100 \%$ ), adherence to this strategy may be better.

Our results also highlight the importance of monitoring patients more often and minimising losses to follow-up. 
Without such effort, increases in viral load go undetected, exposing partners to higher risks of transmission. ${ }^{23}$ With measurement at intervals of 3-12 months, the risk of transmission can be reduced further by basing condom use on measurements taken only 3 months before. This guards against the risk of increases in viral load since the last measurement. ${ }^{25}$ For this reasons, it is important that decisions are not based on viral load measurements taken more than 6 months before.

The model used in this paper incorporates more biological realism than earlier work ${ }^{13} 14$ by using the functional form of the viral load-transmission relationship that reflects a sophisticated analysis of observational data, ${ }^{20}$ explicitly tracking viral load monitoring and modelling the course of viral load evolution over time. Nevertheless, a number of simplifying assumptions were made. The risk of transmission per sex act was scaled to match only an approximate observation of the risk, without considering differences in the frequency of being the insertive or receptive partner. ${ }^{26} 27$ We assumed that, below a certain viral load level, the chance of transmission is low and not actually zero; but evidence is lacking, since observing almost no instances of transmission ${ }^{15}$ could be consistent with either possibility. The response of patients to treatment varies widely and we have attempted to only broadly represent this in the model. However, we believe the key features of viral load evolution have been captured by finding a set of parameters for the viral load trajectory (its rise, period of stability and subsequent rise) and its relationship to treatment adherence. An important limitation of the model is that the effects of other STIs on HIV transmission is not incorporated. The data available from the cohort do not permit a detailed representation of STI transmission and the influence of STIs would depend on many factors, including the pattern of STI spread across the populations. If STIs were included, the overall estimate of HIV transmission risk would probably be greater, but we would expect the key relationship between risk and patterns of condom use to hold or be strengthened (since more frequent screening affords greater opportunity for treating STIs). Finally, the model does not include the influence of intermittent viraemia on the chance of HIV transmission, since we assumed its influence on transmission to be relatively small. ${ }^{28}$

The implications of this work are that the key message to patients should remain that always using condoms when receiving treatment is the best way to protect partners from the risk of HIV transmission. However, an additional message is that using condoms is most crucial when patients have not recently (within the past 3 months) had an undetectable viral load measurement. This message refines the intuitive association between successful treatment and reduced transmission ${ }^{24} 29$ and could substantially improve protection for infected partners.

\section{Key messages}

- Intermittent use of condoms by men receiving treatment offers relatively little reduction in the chance of transmission to their partner.

- In contrast, the chance of HIV transmission can be substantially reduced if condoms are used when the last undetectable viral load measurement was not within the past 3 months.

- Frequent viral load measurement can maximise the potential for treatment to reduce HIV transmission.
This advice must be supported by frequent viral load monitoring (at least every 6 months, but preferably every 3 months) of all patients receiving treatment.

Acknowledgements TBH thanks the Wellcome Trust for funding support. We thank two reviewers for their constructive assessment of this work.

Funding Wellcome Trust.

Competing interests None.

Provenance and peer review Not commissioned; externally peer reviewed.

\section{REFERENCES}

1. Hammer SM, Squires KE, Hughes MD, et al. A controlled trial of two nucleoside analogues plus indinavir in persons with human immunodeficiency virus infection and CD4 cell counts of 200 per cubic millimeter or less. AIDS Clinical Trials Group 320 Study Team. N Engl J Med 1997;337:725-33.

2. Staszewski S, Morales-Ramirez J, Tashima KT, et al. Efavirenz plus zidovudine and lamivudine, efavirenz plus indinavir and indinavir plus zidovudine and lamivudine in the treatment of HIV-1 infection in adults. Study 006 Team. N Engl J Med 1999;341:1865-73.

3. UNAIDS. Report on the Global AIDS epidemic, http://www.unaids.org/en/ knowledge/HIVdata/globalreport/2008/2008 global report.asp, 2008.

4. Jahn A, Floyd S, Crampin AC, et al. Population-level effect of HIV on adult mortality and early evidence of reversal after introduction of antiretroviral therapy in Malawi. Lancet 2008;371:1603-11.

5. When To Start Consortium, Sterne JA, May M, Costagliola D, et al. Timing of initiation of antiretroviral therapy in AIDS-free HIV-1-infected patients: a collaborative analysis of 18 HIV cohort studies. Lancet 2009:373:1352-63.

6. Montaner JS, Hogg R, Wood E, et al. The case for expanding access to highly active antiretroviral therapy to curb the growth of the HIV epidemic. Lancet 2006;368:531-6.

7. Granich RM, Gilks CF, Dye C, et al. Universal voluntary HIV testing with immediate antiretroviral therapy as a strategy for elimination of HIV transmission: a mathematical model. Lancet 2009:373:48-57.

8. Quinn TC, Wawer MJ, Sewankambo N, et al. Viral load and heterosexual transmission of human immunodeficiency virus type 1. Rakai Project Study Group. N Engl J Med 2000;342:921-9.

9. Vernazza P, Hirschel B, Bernasconi E, et al. Les personnes séropositives ne souffrant d'aucune autre MST et suivant un traitement antirétroviral efficace ne transmettent pas le VIH par voie sexuelle. Bulletin des médecins suisses 2008;89:165-9.

10. Vernazza PL. The debate continues: does 'undetectable' mean 'uninfectious'? HIV ther 2009;3:113-16.

11. National Association of People Living With HIVIAIDS 2008. AIDS 2008: The Swiss Statement- the debate continues, 2009. Available at http://napwa.org.au/pl/2008/ 09/aids-2008-the-swiss-statement-the-debate-continues (accessed 29 June 2010)

12. Sturmer M, Doerr HW, Berger A, et al. Is transmission of HIV-1 in non-viraemic serodiscordant couples possible? Antivir Ther 2008:13:729-32.

13. Wilson DP, Law MG, Grulich AE, et al. Relation between HIV viral load and infectiousness: a model-based analysis. Lancet 2008;372:314-20.

14. Garnett GP, Gazzard B. Risk of HIV transmission in discordant couples. Lancet 2008;372:270-1

15. Attia S, Egger M, Muller $M$, et al. Sexual transmission of HIV according to viral load and antiretroviral therapy: systematic review and meta-analysis. Aids 2009:23:1397-404.

16. Wilson DP. Data are lacking for quantifying HIV transmission risk in the presence of effective antiretroviral therapy. Aids 2009;23:1431-3.

17. van Sighem Al, van de Wiel MA, Ghani AC, et al. Mortality and progression to AIDS after starting highly active antiretroviral therapy. Aids 2003;17:2227-36.

18. Hallett TB, Gregson S, Dube S, et al. The impact of monitoring HIV patients prior to treatment in resource-poor settings: insights from mathematical modelling. PLoS Med 2008;5:e53

19. Smit C, Hallett TB, Lange J, et al. Late entry to HIV care limits the impact of antiretroviral therapy in The Netherlands. PLoS One 2008;3:e1949.

20. Fraser C, Hollingsworth TD, Chapman R, et al. Variation in HIV-1 set-point viral load: epidemiological analysis and an evolutionary hypothesis. Proc Natl Acad Sci U S A 2007:104:17441-6.

21. Braithwaite RS, Justice AC, Chang CC, et al. Estimating the proportion of patients infected with HIV who will die of comorbid diseases. Am J Med 2005:118:890-8.

22. Centers for Disease Control and Prevention. http://www.cdc.gov/hiv/, 2008

23. Ndiaye B, Ould-Kaci K, Salleron J, et al. Characteristics of and outcomes in HIV-infected patients who return to care after loss to follow-up. AIDS 2009;23:1786-9

24. Stolte IG, de Wit JB, van EA, et al. Perceived viral load, but not actual HIV-1-RNA load, is associated with sexual risk behaviour among HIV-infected homosexual men. AIDS 2004:18:1943-9.

25. Hirschel B. Meal. How reliable is an undetectable viral load (VL)? The past as a guide to the future. XVII International AIDS conference. Mexico City: 2008 
26. Vittinghoff $\mathbf{E}$, Douglas J, Judson $\mathrm{F}$, et al. Per-contact risk of human immunodeficiency virus transmission between male sexual partners. Am J Epidemiol 1999:150:306-11.

27. Koblin BA, Husnik MJ, Colfax G, et al. Risk factors for HIV infection among men who have sex with men. Aids 2006;20:731-9.
28. Podsadecki TJ, Vrijens BC, Tousset EP, et al. Decreased adherence to antiretroviral therapy observed prior to transient human immunodeficiency virus type 1 viremia. J Infect Dis 2007;196:1773-8.

29. Wasserfallen F, SRWH. Swiss Statement for PLWHA on effective ARV treatment XVII International AIDS conference. Mexico City: 2008.

\section{Book review}

\section{Introduction to Infectious Disease Modelling}

Edited by Emilia Vynnycky, Richard White. Published by Oxford University Press, Oxford, paperback, June 2010 £32.95 (soft cover), pp 368. ISBN 13: 9780198565765 ISBN 10: 0198565763

Mathematical models play an increasingly important role in our understanding of the epidemiology and control of infectious disease. An Introduction to Infectious Disease Modelling by Vynnycky and White aims to equip its readers with the knowledge and skills to develop and use their own models. The content draws on the authors' extensive experience teaching and developing the successful short course and MSc module at the London School of Hygiene and Tropical Medicine. The book is especially successful in its ambition to be accessible to nonmathematicians, including carefully worked step-by-step examples with clear explanations of the mathematical concepts as well as a useful 'Basic maths' reference section. The authors also use their own research experience to provide contemporary, real-life examples of the application of the different types of models described. The online resource of models and examples is an invaluable addition to the text content (http://anintro ductiontoinfectiousdiseasemodelling.com/).

The book is divided into a logical sequence of chapters. After an introduction by Paul Fine, there are chapters discussing what a model is and how to set one up (2/3) and the dynamics that make infectious disease models so interesting (chapter 4). Next they consider some extensions of the simple models to include age structure (chapter 5) and stochastic effects (chapter 6). In chapter 7 , the authors consider in more detail contact patterns between individuals that result in disease transmission. Chapter 8 considers models of curable sexually transmitted infections (STIs) and HIV. Chapter 9 discusses some special topics including the effect of STI coinfection on HIV transmission. Each chapter has clearly stated aims and panels containing more detailed information that can be skipped through until required, which makes it easy to navigate through to the most relevant pieces of information. This book nicely fills in both the philosophical and technical details between the concept of mathematical modelling and its application.

This introduction bridges the mathematical gaps left by the classic Infectious Diseases of Humans by Anderson and May, as well as providing an update on methods and recent examples of model applications. Also recently published is Modelling Infectious Diseases in Humans and Animals by Keeling and Rohani (http://www.modelinginfectiousdiseases.org). This shares a similar format of providing detailed mathematical descriptions, online resources and model code. Inevitably, there is a fair amount of overlap, but the diseases and examples used are rather different. However, both are excellent, and the choice will likely come down to disease and preferred modelling environment. Vynnycky and White online models are provided in Excel and
Berkeley Madonna rather than source code (Fortran, C or Matlab). For the readers of Sexually Transmitted Infections, Vynnycky and White is the clear choice for their comprehensive content on STI and HIV models.

This is an excellent text book and readers can be assured that 'it does what it says on the tin': provide a thorough introduction to infectious disease modelling. This book will be very useful to Masters and $\mathrm{PhD}$ students throughout their courses. I would certainly have found it very useful during my doctorate, especially as I do not have a background in engineering or maths. It will also be of interest to more experienced researchers, clinicians and policy makers who wish to gain a better understanding, as mathematical models play an increasingly important role in guiding control policy and interventions against infectious diseases. I will be turning to An Introduction to Infectious Disease Modelling for reference frequently for its clear explanations and topical worked examples. I recommend this book very highly to new students and more experienced colleagues alike.

Katy Turner

Correspondence to Katy Turner, School of Social and Community Medicine, University of Bristol, UK; katy. turner@bristol.ac.uk

Provenance and peer review Commissioned; not externally peer reviewed.

Published Online First 8 November 2010

Sex Transm Infect 2011;87:21.

doi:10.1136/sti.2010.046342 